\title{
ALCANCE DEL PRINCIPIO DE PARTICIPACIÓN EQUILIBRADA DE GÉNERO EN EL MARCO JURÍDICO INTERNACIONAL: NACIONES UNIDAS, UNIÓN INTERPARLAMENTARIA, CONSEJO DE EUROPA Y UNIÓN EUROPEA
}

\section{Scope of the principle of gender-balanced participation in the international legal framework: United Nations, Inter-Parliamentary Union, Council of Europa and European Union}

\author{
María Nieves Saldaña \\ manieves@uhu.es \\ Universidad de Huelva - España
}

Recibido: 28-02-2019

Aceptado: 10-05-2019

\section{Resumen}

El reconocimiento de la participación equilibrada de género como principio para hacer efectiva la igualdad de mujeres y hombres y así legitimar los sistemas democráticos se ha llevado a cabo durante los últimos veinte años a través de diversos acuerdos de escasa fuerza vinculante (soft law) de las Naciones Unidas, la Unión Interparlamentaria, el Consejo de Europa y la Unión Europea. De ahí que se mantenga la infrarepresentación de las mujeres en todos los ámbitos y niveles decisorios, siendo necesario que se implemente a través de instrumentos jurídicos vinculantes que obliguen a las propias organizaciones internacionales a su efectiva aplicación y a los Estados miembros a su reconocimiento y regulación con el máximo rango normativo, a nivel constitucional y legal.

Palabras clave: Participación equilibrada de género, Naciones Unidas, Unión Interparlamentaria, Consejo de Europa, Unión Europea.

\begin{abstract}
The recognition of gender-balanced participation as a principle to make effective the equality of women and men and thus legitimize democratic systems has been carried out during the last twenty years through various agreements of low binding force (soft law) of the United Nations, the InterParliamentary Union, the Council of Europe and the European Union. Hence, the underrepresentation of women in all areas and levels of decision-making is maintained, being necessary to implement it through binding legal instruments that oblige the international organizations themselves to effectively apply them and the member states to recognize and regulate them with the maximum normative range, at the constitutional and legal level.
\end{abstract}

Keywords: Gender-balanced participation, United Nations, Inter-Parliamentary Union, Council of Europe, European Union. 


\section{Introducción. La participación equilibrada de género en el marco jurídico internacional: de la "masa crítica" al "50/50"}

La participación equilibrada de género (gender-balanced participation) hace referencia al porcentaje de participación necesario para que pueda hablarse de una participación efectiva de las mujeres en el proceso de toma de decisiones y de cambio en las estructuras de poder (Moss Kanter, 1977). Este porcentaje fue establecido por las Naciones Unidas en Beijing con carácter transversal en la llamada "masa crítica", esto es, un 30\% de mujeres en todos los ámbitos de la toma de decisiones y a todos los niveles (Naciones Unidas, 1996). Recientemente, los acuerdos del Consejo de Europa (Council of Europe, 2003) y la Unión Europea (Unión Europea, 1996) han incrementado el porcentaje de participación de las mujeres hasta el $40 \%$ para que pueda hablarse realmente de un equilibrio de género en la composición de los órganos decisorios. Aunque, en sentido estricto, la participación equilibrada de género implica una participación equitativa, lo que supone una participación de mujeres y hombres paritaria (50\%).

Sin embargo, no se trata de un asunto meramente cuantitativo, de elevar el número de mujeres en los puestos decisorios, es fundamentalmente de carácter cualitativo, de la calidad y mejora de los procesos decisorios en los sistemas democráticos y, definitivamente, de justicia hacia la mitad del género humano, del inalienable derecho a decidir sobre todo aquello que afecta a su propia existencia. Así, entre los argumentos que se esgrimen para su justificación, se señala que si la participación de las mujeres alcanza entre el 30 y 35\% pueden tener verdaderas repercusiones en el estilo político y en el contenido de las decisiones y la renovación de la vida política (Naciones Unidas, 1997); que la participación equilibrada de las mujeres y los hombres en la toma de decisión política y pública forma parte del pleno disfrute de los derechos humanos y constituye un elemento de justicia social y una condición necesaria para un mejor funcionamiento de una sociedad democrática (Consejo de Europa, 2003); y que una participación equilibrada en la toma de decisiones en cada ámbito de la vida constituye una importante condición para la igualdad entre hombres y mujeres (Unión Europea, 1995a).

De ahí que a lo largo de estos años, la exigencia de una participación equilibrada de género en la toma de decisiones se haya extendido del ámbito estrictamente político, referido fundamentalmente a los órganos legislativos y otros órganos elegidos democráticamente en elecciones locales, regionales, nacionales y supranacionales, al ámbito público, a los órganos decisorios de carácter gubernamental y comités públicos de nombramiento ministerial o gubernamental, incluidas las delegaciones nacionales en los organismos y foros internacionales, transitando finalmente a la esfera privada, a la toma de decisiones en los ámbitos económico y social, esto es, en los puestos de responsabilidad y decisión de las organizaciones empresariales y sindicales, colegios profesionales, asociaciones y medios de comunicación, incluidos los órganos decisorios de los partidos políticos. 
En el marco jurídico internacional, la consolidación de la participación equilibrada de género como principio para hacer efectiva la igualdad de mujeres y hombres y así legitimar los sistemas democráticos tiene lugar en los textos más recientes adoptados por las Naciones Unidas, y la Unión Interparlamentaria, así como por el Consejo de Europa y la Unión Europea. En efecto, aunque las Naciones Unidas han promovido la igualdad política y pública de mujeres y hombres desde sus primeros Tratados y Acuerdos internacionales, sin embargo, la Declaración y Plataforma de Acción de Beijing adoptadas en 1995 inauguran una nueva etapa al incorporarse la perspectiva transversal de la igualdad de género en todos las políticas (gender mainstreaming), constituyendo un importante avance para la participación equilibrada en el proceso de toma de decisiones, al concretarse el objetivo de alcanzar un equilibrio de un 30\% de mujeres en puestos de representación y decisorios.

Asimismo, para la superación de la infrarepresentación de las mujeres en los Parlamentos nacionales a nivel mundial, la Unión Interparlamentaria (Inter-Parliamentary Union, IPU) ha adoptado una serie de documentos y ha diseñado numerosas estrategias en programas de acción específicos para corregir los desequilibrios de género en los órganos parlamentarios. De igual manera, la participación equilibrada de género en todos los procesos decisorios está presente en los textos más recientes aprobados por los órganos del Consejo de Europa, especialmente el Comité de Ministros, la Asamblea Parlamentaria y el Congreso de Poderes Locales y Regionales, para garantizar la igual participación de mujeres y hombres en todos los ámbitos de poder. Y en el marco de la Unión Europea, la exigencia de la participación equilibrada de mujeres y hombres en los procesos decisorios se ha extendido progresivamente a todos los ámbitos de la toma de decisiones de los Estados miembros y de las propias instituciones europeas, promoviéndose la paridad de género, esto es, 50/50 de cada sexo, para las elecciones al Parlamento Europeo que tienen lugar en mayo de 2019 (European Women's Lobby, 2018).

\section{La participación equilibrada de género en las Naciones Unidas: desde Beijing a los objetivos de desarrollo sostenible}

Aunque las Naciones Unidas han promovido la igualdad política y pública de mujeres y hombres desde sus primeros Tratados y Acuerdos internacionales (Saldaña Díaz, 2010), sin embargo, la Declaración y Plataforma de Acción de Beijing, adoptadas en la IV Conferencia Mundial de las Naciones Unidas sobre las mujeres, celebrada del 4 al 15 de septiembre de 1995, inauguran una nueva etapa al incorporarse la perspectiva transversal de la igualdad de género en todos las políticas (gender mainstreaming), constituyendo un importante avance para la participación equilibrada en el proceso de toma de decisiones, al concretarse el objetivo de alcanzar la llamada "masa crítica", esto es, un 30\% de mujeres en todos los ámbitos de la toma de decisiones 
y a todos los niveles, extendiéndose en el Objetivo Estratégico G, "La mujer en el ejercicio del poder y la adopción de decisiones", la exigencia de participación equilibrada de género a todos los niveles de las estructuras de poder y a todos los procesos decisorios, tanto públicos como privados: sistema electoral, órganos y comités gubernamentales, entidades de la administración pública, judicatura, representación internacional, estructuras internas de los partidos políticos, sindicatos, organizaciones de empleadores, medios de comunicación, instituciones de investigación y académicas, asociaciones, organizaciones no gubernamentales e internacionales (Naciones Unidas, 1996: 85-91).

Igualmente, la Recomendación General 23 sobre la "Vida política y pública" adoptada por el Comité para la Eliminación de la Discriminación contra la Mujer en 1997, postula la masa crítica de mujeres en todos los órganos decisorios, especialmente en las negociaciones internacionales, las actividades de mantenimiento de la paz, en todos los ámbitos de la diplomacia preventiva, la mediación, la reconciliación social, las negociaciones de paz, el sistema internacional de justicia penal y en todos los órganos de las Naciones Unidas, entre ellos las Comisiones Principales de la Asamblea General, el Consejo Económico y Social y los órganos de expertos, en particular los órganos creados en virtud de tratados, así como en el nombramiento de grupos de trabajo independientes o de relatores especiales o por países (Naciones Unidas, 1997: 16-40).

Tres años más tarde, en el Informe del Comité Especial Plenario del vigésimo tercer periodo extraordinario de sesiones de la Asamblea General de las Naciones Unidas celebrado bajo el lema "La mujer en el año 2000: igualdad entre los géneros, desarrollo y paz para el siglo XXI" (Beijing+5) en Nueva York del 5 al 9 de junio de 2000, que examina y evalúa los progresos alcanzados y propone nuevas medidas e iniciativas para superar los obstáculos en la aplicación de la Plataforma de Acción de Beijing, en relación con el Objetivo Estratégico G, "La mujer en el ejercicio del poder y la adopción de decisiones", la participación equilibrada de género alcanza a todos los ámbitos de la vida pública, especialmente a los puestos de adopción de decisiones y formulación de políticas, a los partidos políticos, a todos los ministerios gubernamentales y a las principales instituciones encargadas de la formulación de políticas, así como a los órganos y entidades locales de desarrollo (Naciones Unidas, 2000: 16-17, 28 y 43).

Asimismo, en el Informe Final sobre el 50 período de sesiones de la Comisión de la Condición Jurídica y Social de la Mujer de las Naciones Unidas (22 de marzo de 2005, 27 de febrero a 10 de marzo y 16 de marzo de 2006), en las Conclusiones Convenidas sobre la "Participación de las mujeres y los hombres en condiciones de igualdad en los procesos de adopción de decisiones a todos los niveles", la exigencia de una participación equilibrada de mujeres y hombres en los procesos decisorios alcanza su máxima transversalidad, afectando a todos los niveles y en todos los ámbitos y esferas directivas, especialmente las de la política macroeconómica, el comercio, el trabajo, los presupuestos, la defensa y las relaciones exteriores, los medios de comunicación, el poder judicial y órganos de gestión electoral, sin olvidar los procesos de 
consolidación de la paz, reconstrucción, rehabilitación y reconciliación después de los conflictos (Naciones Unidas, 2006: 31-38).

Igualmente, la Resolución A/RES/62/137, de 18 de diciembre de 2007, de la Asamblea General de las Naciones Unidas, sobre el "Seguimiento de la Cuarta Conferencia Mundial sobre la Mujer y plena aplicación de la Declaración y la Plataforma de Acción de Beijing y de los resultados del vigésimo tercer período extraordinario de sesiones de la Asamblea General", sigue exhortando a los gobiernos y a los fondos y programas y órganos y organismos especializados competentes del sistema de las Naciones Unidas, en el marco de sus respectivos mandatos, y se invita a las instituciones financieras internacionales y a todas las instancias pertinentes de la sociedad civil, incluidas las organizaciones no gubernamentales, a que intensifiquen sus iniciativas y medidas para asegurar la plena representación y participación plena e igual de la mujer en la toma de decisiones políticas, sociales y económicas, como condición esencial para la igualdad de género (Naciones Unidas, 2007: punto 7).

Y, más recientemente, la “Agenda 2030 para el Desarrollo Sostenible “, aprobada en la Cumbre de las Naciones Unidas para el Desarrollo Sostenible en octubre de 2015, que elabora un nuevo modelo para el desarrollo mundial, hace hincapié en que lograr la igualdad de género y el empoderamiento de las mujeres contribuirá decisivamente al logro de todos los Objetivos de Desarrollo Sostenible (ODS), y en particular el Objetivo 5.5 se dirige a garantizar la participación plena y efectiva de las mujeres y la igualdad de oportunidades de liderazgo en todos los niveles de decisión en la esfera política, económica y pública (Naciones Unidas, 2015: 20).

\section{La participación equilibrada de género en la Unión Interparlamentaria: de la alianza entre hombres y mujeres en política a la libertad de las mujeres de participar plenamente en los procesos políticos de manera segura y sin interferencias}

Para la superación de la infrarepresentación de las mujeres en los Parlamentos nacionales a nivel mundial, la Unión Interparlamentaria (UIP) (Inter-Parliamentary Union, IPU), organización internacional que integra a más de 160 Parlamentos nacionales, ha promocionado a lo largo de los últimos 40 años la participación de las mujeres en el proceso de toma de decisiones, especialmente en los Parlamentos, considerando la participación de las mujeres en la vida política un elemento esencial de la democracia. Para su consecución, la UIP ha venido sistematizando los datos estadísticos referidos a la proporción de mujeres en los Parlamentos nacionales en estudios comparativos a nivel mundial, ha diseñado estrategias integrales para superar la infrarepresentación de las mujeres y el actual desequilibrio de género que afecta a los Parlamentos nacionales y a la propia UIP y ha adoptado en 2012 un Plan de Acción para los Parlamentos sensibles al género (Saldaña Díaz, 2014). 
Así, la Unión Interparlamentaria promueve ya la masa crítica del 30\% de mujeres en la 105 Conferencia Interparlamentaria Especial que con el título "Hacia una alianza entre hombres y mujeres en política" se celebró en Nueva Dehli entre el 14 y el 18 de febrero de 1997, proponiéndose un cupo o cuota del $30 \%$ de mujeres en las listas electorales, contemplándose asimismo la reserva de cierto número de escaños en las instancias representativas nacionales y locales y la colocación de las candidaturas de mujeres en los puestos de las listas que tengan más probabilidades de ser elegidos (Inter-Parliamentary Union, 1997: 14).

Igualmente, la Resolución "Beijing+10: una evaluación desde la perspectiva parlamentaria", aprobada por la $111^{\text {a }}$ Asamblea de la Unión Interparlamentaria el 1 de octubre de 2004, recomienda que haya una mayor presencia de mujeres en las estructuras directivas de los Parlamentos nacionales y los foros interparlamentarios, así como una representación nacional equilibrada de género en las relaciones parlamentarias exteriores, a nivel lateral y multilateral. Asimismo, se recomienda aumentar el número de mujeres que integran las comisiones parlamentarias, procurando alcanzar una meta del 50\%, de conformidad con el documento final aprobado por la Asamblea General de las Naciones Unidas en su período extraordinario de sesiones de 2000, titulado "La mujer en el año 2000: igualdad entre los géneros, desarrollo y paz para el siglo XXI" (Beijing+5), o de un 30\%, como mínimo, de modo que las mujeres puedan influir en los enfoques de la legislación que se prepare, así como incorporar en ella sus diversas perspectivas e inquietudes (InterParliamentary Union, 2004: 5-7).

Posteriormente, la Estrategia de la Unión Interparlamentaria para 2012-2017 denominada “Mejores Parlamentos para democracias más fuertes", aprobada en octubre de 2011, establece como segundo objetivo de la $1^{\mathrm{a}}$ Orientación Estratégica el impulso de la igualdad de género en el ámbito parlamentario, que, centrado en cuatro áreas específicas, incluye el fortalecimiento de los marcos nacionales para facilitar el acceso de las mujeres al Parlamento, contemplándose en su $3^{\text {a }}$ Orientación Estratégica la formulación de directrices sobre el equilibrio de género en las Delegaciones parlamentarias con miras a incrementar la participación de mujeres parlamentarias (Inter-Parliamentary Union, 2011: 5, 10).

Igualmente, el "Plan de Acción para los Parlamentos sensibles al género", aprobado en la $127^{\mathrm{a}}$ Asamblea de la Unión Interparlamentaria, celebrada en Quebec el 26 de octubre de 2012, con el objetivo de apoyar los esfuerzos de los Parlamentos por convertirse en instituciones más sensibles a la igualdad de género, incluye como un área específica de acción incrementar el número de mujeres en el Parlamento y lograr una participación paritaria, proponiéndose para su consecución diversas actuaciones, entre otras: adoptar medidas especiales para que los partidos seleccionen a un mayor número de mujeres para presentarse como candidatas a escaños que tengan posibilidad de ganar, así como proponer una reforma electoral de la Constitución que prevea la asignación de escaños reservados a mujeres; adoptar medidas de acción positiva y modificar el reglamento con objeto de dar preferencia a las mujeres sobre los hombres para desempeñar cargos parlamentarios (incluidas las presidencias de las comisiones y posiciones de liderazgo en la Mesa o la Junta) en los 
casos en que las cualificaciones sean iguales o proporcionales a su representación en el parlamento; rotar las posiciones de liderazgo parlamentario entre hombres y mujeres durante un período de tiempo; promover la distribución proporcional y equitativa de las mujeres parlamentarias en todas las comisiones, y no sólo en las que se ocupan de cuestiones relacionadas con la mujer, el niño, el género, la familia, la salud y la educación (Inter-Parliamentary Union, 2012: 14-15).

La vigente Estrategia de la Unión Interparlamentaria para 2017-2021, intitulada "Parlamentos democráticos fuertes al servicio del pueblo", incluye entre las medidas a adoptar en el Objetivo Estratégico 2, dirigido a promover la igualdad de género y el respeto de los derechos de la mujer, la formulación y el refuerzo de estrategias y marcos nacionales que faciliten el acceso pleno y en condiciones de igualdad de las mujeres a los Parlamentos, incrementando su contribución en la elaboración de las políticas (Inter-Parliamentary Union, 2016a).

Y la Resolución relativa a "La libertad de las mujeres de participar plenamente en los procesos políticos, de manera segura y sin interferencias: La creación de asociaciones entre hombres y mujeres para alcanzar este objetivo", aprobada por la $135^{\mathrm{a}}$ Asamblea de la Unión Interparlamentaria el 26 de octubre de 2016, entre otras muchas medidas, insta encarecidamente a los Parlamentos nacionales a fijar un plazo para que al menos el $30 \%$ de sus miembros sean mujeres y fijen un plazo adicional para que esa proporción alcance el 50\%, adoptando y aplicando sistemas de cuotas u otras medidas similares que permitan que las mujeres sean seleccionadas en posiciones elegibles en las listas (Inter-Parliamentary Union, 2016b:10-11).

\section{La participación equilibrada de género en el Consejo de Europa: de la Recomendación del Comité de Ministros de 2003 a la Estrategia de Igualdad de Género 2018-2023}

La participación equilibrada de género en todos los procesos decisorios está presente en los textos más recientes aprobados por los órganos del Consejo de Europa, especialmente el Comité de Ministros, la Asamblea Parlamentaria y el Congreso de Poderes Locales y Regionales (Saldaña Díaz, 2013). Así, la Recomendación Rec(2003)3 del Comité de Ministros a los Estados Miembros relativa a la "Participación equilibrada de las mujeres y los hombres en los procesos de toma de decisión en los ámbitos político y público”, de 12 de marzo de 2003, propone considerar posibles cambios constitucionales y/o legislativos, incluidas medidas de acción positiva, que pudieran favorecer una participación más equilibrada de género en la toma de decisión política y pública, que tengan como objetivo alcanzar el $40 \%$ de ambos sexos en los puestos de representación y decisión como umbral de paridad para eliminar posibles sesgos de género en los procesos de toma de decisiones, especialmente en los siguientes ámbitos: en las candidaturas a las elecciones locales, regionales, nacionales y supranacionales; en todos los cargos de comités públicos de nombramiento ministerial o gubernamental; en las delegaciones nacionales a organismos y foros internacionales; 
en los puestos de decisión dentro de las estructuras de los partidos; en los puestos de responsabilidad y decisión de las organizaciones empresariales y sindicales; en los órganos de decisión de empresas y asociaciones, especialmente en aquellas subvencionadas para la prestación de servicios públicos o la aplicación de políticas públicas; y en los puestos de decisión de los medios de comunicación (Council of Europe, 2003: 3 y ss).

Dos años más tarde, la Resolución 1366 (2004) de la Asamblea Parlamentaria relativa a "Los candidatos para el Tribunal Europeo de Derechos Humanos", de 30 de enero de 2004 (Council of Europe, 2004: 3.ii), con la modificación introducida por la Resolución 1426 (2005), de 18 de marzo de 2005, aprueba no tener en cuenta las listas de candidatos que no incluyan, al menos, uno de cada sexo, excepto cuando pertenezcan al sexo infrarepresentado en el Tribunal, esto es, que representen menos del 40\% del número total de miembros (Council of Europe 2005: 5).

Posteriormente, en la Resolución 1585 (2007) de la Asamblea Parlamentaria sobre "Los principios de igualdad de género en la Asamblea Parlamentaria”, de 23 de noviembre de 2007, se urge a los Parlamentos nacionales de los Estados miembros a asegurar que las mujeres estén presentes en las delegaciones nacionales al menos en la misma proporción en que lo están en los Parlamentos nacionales, con el objetivo de alcanzar, como mínimo, el 30\%. Asimismo, para implementar el equilibrio de género en el ámbito de la presidencia y las vicepresidencias, se modifican las normas de procedimiento de la Asamblea incorporándose en la norma 48.7, relativa a la elección de la presidencia y vicepresidencia de los Subcomités, que se tenga en cuenta el principio de igualdad de género (Council of Europe, 2007: 9, 15.3, 45.1, 48.7 y 49.1).

Asimismo, en la Resolución 1706 (2010) de la Asamblea Parlamentaria relativa a "El aumento de la representación de las mujeres en la política a través del sistema electoral”, de 27 de enero de 2010, se constata que las mujeres siguen infrarepresentadas, instándose a los Estados miembros a rectificar esta situación con carácter prioritario, especialmente: vinculando las previsiones sobre igualdad de género y no discriminación recogidas en las Constituciones y en la legislación electoral con la adopción de medidas de acción positiva a favor del sexo menos representado; reformando el sistema electoral a uno más favorable a la representación de las mujeres en el Parlamento, introduciéndose, en el caso de países con un sistema de listas de representación proporcional, una cuota que permita no sólo una elevada proporción de mujeres candidatas, preferiblemente al menos el $40 \%$, sino incluso un "sistema cremallera" de alternancia de los candidatos de uno y otro sexo, rechazándose las candidaturas en caso de incumplimiento, y en el caso de países con sistemas de mayoría o plurales, adoptando el principio de que cada partido elija a la persona candidata entre al menos un hombre y una mujer nominados en cada distrito del partido, o implementando cuotas de género obligatorias en el seno de los partidos, con aplicación de sanciones efectivas en caso de incumplimiento (Council of Europe, 2010: 6).

De igual manera, en la Resolución 1825 (2011) de la Asamblea Parlamentaria relativa a "Un número mayor de mujeres en los órganos de la toma de decisiones económica y social", de 23 de noviembre de 2011, se insta a los Estados miembros del Consejo de Europa, entre otras medidas, a: 
adoptar una legislación que requiera que las instituciones públicas y privadas alcancen una representación mínima del $40 \%$ de mujeres en puestos directivos y de toma de decisiones dentro de un plazo claramente definido, poniendo en marcha los mecanismos necesarios para controlar la aplicación de dicha legislación; y a introducir la obligación de que las empresas de propiedad estatal y las grandes compañías garanticen una representación mínima del 40\% de mujeres en sus consejos de gobierno y administración (Council of Europe, 2011: 7.11-7.13, 8.5).

En el mismo sentido, en la Resolución 1898 (2012) de la Asamblea Parlamentaria sobre "Partidos políticos y representación política de las mujeres", de 3 de octubre de 2012, se insta a introducir un cupo mínimo del $40 \%$ del sexo menos representado en los órganos ejecutivos de toma de decisiones de los partidos políticos a todos los niveles; en el caso de sistemas electorales proporcionales, introducir una cuota mínima del $40 \%$ de personas del sexo menos representado en las listas electorales, acompañada de garantías especiales de género en cuanto al orden de clasificación y a las posiciones en la parte superior de la lista, preferiblemente a través de un sistema cremallera; y en el caso de sistemas electorales mayoritarios, fomentar la selección de candidatos del sexo menos representado a través de candidaturas preseleccionadas compuestas exclusivamente por mujeres (all-women shortlists) o listas prioritarias con un número igual de personas de ambos sexos (Council of Europe, 2012: 6, 7).

Posteriormente, en la "Estrategia de Igualdad de Género 2014-2017", aprobada por el Comité de Ministros el 6 de noviembre de 2013, figura como cuarto objetivo estratégico lograr una participación equilibrada de mujeres y hombres en la toma de decisiones política y pública que no sea inferior al 40\% para ambos sexos, relacionándose diversas acciones para su consecución tanto en los Estados miembros como en los órganos del propio Consejo de Europa (Council of Europe, 2013). Tres años más tarde, en la Resolución 2111 (2016) de la Asamblea Parlamentaria, de 21 de abril de 2016, relativa a la "Evaluación del impacto de las medidas para mejorar la representación política de las mujeres", se insta a los Estados miembros del Consejo de Europa a que consideren la introducción del principio de la paridad en la Constitución o en la legislación nacional, a que introduzcan en la legislación electoral mandatos de colocación estrictos o parejas de candidatos del género opuesto, y a que organismos independientes, como tribunales o comisiones electorales, supervisen la aplicación de cuotas y apliquen sanciones, afirmándose que la Asamblea apoya el principio de paridad de género, que representa el objetivo último de la representación política, cuya aplicación requiere que el Estado vaya más allá de las medidas positivas y garantice una representación equitativa de mujeres y hombres en los órganos elegidos y en otras instituciones a todos los niveles (Council of Europe, 2016a: 15, 16).

Igualmente, en la Recomendación 390(2016) del Congreso de Poderes Locales y Regionales del Consejo de Europa, relativa a la "Participación política de las mujeres a nivel local y regional", de 20 de octubre de 2016, se insta al Comité de Ministros a alentar a los gobiernos de los Estados miembros a implementar la Recomendación del Comité de Ministros (2003)3, apoyando a las autoridades locales y regionales en la promoción y aplicación de medidas específicas con miras a 
aumentar la participación política de las mujeres para cumplir con el umbral del $40 \%$ adoptándose reformas legislativas que apliquen cuotas a las candidaturas, con sanciones pecuniarias en caso de incumplimiento (Council of Europe, 2016b).

Y recientemente, en la "Estrategia de Igualdad de Género 2018-2023", aprobada por el Comité de Ministros el 7 de marzo de 2018, se mantiene como cuarto objetivo estratégico conseguir una participación equilibrada de mujeres y hombres en la toma de decisiones tanto políticas como públicas que no sea inferior al $40 \%$ para ambos sexos, relacionándose diversas acciones para su consecución tanto en los Estados miembros como en los órganos del propio Consejo de Europa (Council of Europe, 2018).

\section{La participación equilibrada de género en la Unión Europea: de la Cumbre Europea "Mujeres en el Poder" al Compromiso estratégico para la igualdad entre mujeres y hombres 2016-2019}

En el proceso de configuración internacional de la participación equilibrada de género resulta significativa la contribución de la Unión Europea, especialmente a partir de la Declaración Final adoptada en la I Cumbre Europea "Mujeres en el Poder", celebrada en Atenas el 3 de noviembre de 1992, donde se denunció el déficit de legitimación democrática de los sistemas políticos europeos y se proclamó la inmediata y total integración de las mujeres en las esferas política y pública para su efectiva superación, extendiéndose progresivamente la exigencia de la participación equilibrada de género en los procesos decisorios a todos los ámbitos de la toma de decisiones de los Estados miembros y de las propias instituciones europeas (Saldaña Díaz, 2006).

Tres años más tarde, la Recomendación del Consejo 96/694/E, de 2 de diciembre de 1996, relativa a la "Participación equilibrada de las mujeres y los hombres en los procesos de toma de decisión", recomienda a los Estados miembros promover la participación equilibrada de la mujer en los órganos y comisiones gubernamentales a todos los niveles; en los puestos públicos electivos y no electivos a todos los niveles, prestando especial atención al fomento de una composición equilibrada de los comités, las comisiones y los grupos de trabajo, tanto a escala nacional como a escala comunitaria y en las comisiones que elaboran las pruebas de los concursos y oposiciones, así como de los tribunales que las presiden. En cuanto a las instituciones comunitarias, la Recomendación reclama la participación equilibrada de género en cada institución, órgano y organismo descentralizado de las Comunidades Europeas (Unión Europea, 1996: 11-15).

Asimismo, el 18 de febrero de 1999 la Comisión Europea da pasos concretos para alcanzar la participación equilibrada al adoptar la Comunicación de la Comisión "Mujeres y Ciencia Movilizar a las mujeres en beneficio de la investigación europea", fijándose un objetivo de un $40 \%$ 
de participación femenina en los grupos de expertos, comités consultivos y programas de becas del Quinto Programa Marco de la Comunidad Europea para acciones de investigación, demostración y desarrollo tecnológicos (Unión Europea, 1999). Un año más tarde, el segundo ámbito específico de la Estrategia Marco Comunitaria sobre igualdad entre mujeres y hombres (2001-2005) extiende el objetivo de mejorar el equilibrio de género a la toma de decisiones políticas, a la toma de decisiones socioeconómicas y a la propia Comisión Europea (Unión Europea, 2000a: 7-10). Para su consecución en el ámbito de la Comisión, se adoptó inmediatamente la Decisión de la Comisión, de 27 de junio de 2000, relativa al "Equilibrio entre hombres y mujeres en los Comités y Grupos de Expertos establecidos por la Comisión", en la que se fija en un 40\% el nivel mínimo de participación de personas de cada sexo en sus comités y grupos de expertos, tanto los de nueva creación como los ya existentes, instándose a los Estados miembros a que promuevan el equilibrio entre mujeres y hombres en sus candidaturas para estos órganos (art. 2) (Unión Europea, 2000b).

Igualmente, la Resolución del Parlamento Europeo, de 18 de enero de 2001, sobre el "Informe de la Comisión sobre la aplicación de la Recomendación 96/694 del Consejo de 2 de diciembre de 1996, relativa a la participación equilibrada de las mujeres y los hombres en los procesos de toma de decisión", demanda que se favorezca el equilibrio de género en todas las políticas y en todas las comisiones, tanto a escala de la Unión Europea como a escala nacional e internacional, con una participación no inferior al $40 \%$ para cada sexo, alcanzando la exigencia de participación equilibrada a las mismas instituciones comunitarias, proponiéndose que los Estados miembros incluyan enmiendas al Tratado con el objetivo de fomentar el equilibrio entre los géneros en las instituciones de la Unión Europea (Unión Europea, 2001: 251).

Posteriormente, la Resolución del Parlamento Europeo, de 21 de mayo de 2008, sobre "La mujer y la ciencia", pone de manifiesto la infrarepresentación de las mujeres en los altos niveles de la toma de decisiones de los ámbitos universitarios y de investigación y critica el objetivo de la Unión Europea poco ambicioso e insuficiente de alcanzar el 25\% de mujeres en puestos de liderazgo en la investigación del sector público, recordando a la Comisión y a los Estados miembros que la paridad de género implica al menos el $40 \%$ de mujeres, por lo que pide a la Comisión y a los Estados miembros que establezcan la obligación de garantizar el equilibrio entre hombres y mujeres en los grupos de evaluación y comités de selección y otros consejos, con el objetivo no vinculante de al menos un $40 \%$ de mujeres, y que la propia Comisión vele por que en las licitaciones convocadas en el marco del Séptimo Programa Marco se valore positivamente la representación equilibrada de hombres y mujeres, instándose a los Estados miembros a que tomen las mismas disposiciones en relación con sus planes nacionales y regionales (Unión Europea, 2008: 15-17).

Cuatro años más tarde, en la Resolución del Parlamento Europeo, de 13 de marzo de 2012, relativa a "La representación de las mujeres en los procesos de toma de decisiones políticas: calidad e igualdad", se considera alarmante la infrarepresentación de las mujeres en el Parlamento Europeo, pues aunque ha aumentado al 35\%, sin embargo, no ha alcanzado aún la paridad, teniendo incluso 
las mujeres aún menos representación en los puestos directivos de las comisiones parlamentarias y los grupos políticos, instándose al Consejo, a la Comisión y a los Estados que adopten una serie de medidas y acciones concretas destinadas a incrementar la participación de las mujeres en las elecciones al Parlamento Europeo de 2014, considerándose que el procedimiento para elaborar las listas electorales mediante el cual las candidatas alternan con los candidatos en el primer puesto de las listas es el mejor medio para mejorar la participación de las mujeres en la política y destacándose la necesidad de adoptar medidas concretas destinadas a lograr la paridad en los cargos electivos en los Parlamentos nacionales y en el Parlamento Europeo (como los de presidencia, vicepresidencia y presidencias y vicepresidencias de comisión), por ejemplo estableciendo un objetivo del $50 \%$ en la representación de hombres y las mujeres en cada uno de estos cargos (Unión Europea, 2012a).

Ese mismo año, el 4 de noviembre de 2012 la Comisión adopta una avanzada propuesta de Directiva del Parlamento Europeo y del Consejo destinada a mejorar el equilibrio de género entre los administradores no ejecutivos de las empresas cotizadas y por la que se establecen medidas afines, estableciéndose un objetivo mínimo de un $40 \%$ de presencia del sexo menos representado entre los consejeros no ejecutivos, que debe alcanzarse en 2020 para las empresas cotizadas en bolsa y en 2018 para las empresas públicas cotizadas (Unión Europea, 2012b).

Actualmente, el vigente "Compromiso estratégico para la igualdad entre mujeres y hombres 2016-2019”, adoptado por la Comisión el 3 de diciembre de 2015, establece como área temática prioritaria promover la igualdad entre mujeres y hombres en la toma de decisiones, señalándose entre sus objetivos lograr un mayor equilibrio entre sexos en los puestos de liderazgo económico, en concreto una representación mínima del $40 \%$ del sexo infrarepresentado entre los administradores no ejecutivos de las empresas cotizadas, así como la adopción de medidas para atajar la sobrerrepresentación de los hombres en los puestos decisorios en las organizaciones de investigación y promover el equilibrio en la toma de decisiones políticas y la vida pública, incluyéndose el 40\% de mujeres en puestos de nivel directivo superior y medio para finales de 2019 en la propia Comisión Europea (Unión Europea, 2016: 27-28).

Y la reciente Resolución del Parlamento Europeo, de 15 de enero de 2019, relativa a la "Integración de la perspectiva de género en el Parlamento Europeo", insta a los partidos políticos europeos a garantizar una representación equilibrada de género de las candidaturas para las elecciones al Parlamento Europeo en 2019 mediante la presentación de "listas cremallera" o paritarias; hace un llamamiento a los grupos políticos para que de cara a la legislatura de 2019-2024 garanticen una composición equilibrada de género de los órganos de gobierno del Parlamento Europeo, recomendando que presenten a miembros masculinos y femeninos para los cargos de presidencia, vicepresidencia y miembros de la Mesa, así como para las presidencias de los comités y delegaciones; y recomienda que para la legislatura de 2019-2024 los grupos políticos del Parlamento Europeo elijan a dos miembros, un hombre y una mujer, para el cargo de la copresidencia del grupo (Unión Europea, 2019). 


\section{Conclusiones. Limitaciones y déficits del principio de participación equilibrada de género en el marco jurídico internacional: propuestas para garantizar su carácter vinculante}

Aunque la igual participación de mujeres y hombres en la toma de decisiones es una cuestión de justicia, derechos humanos y de legitimación democrática, y a pesar del cúmulo de resoluciones, recomendaciones y acuerdos internacionales que, como se ha analizado detenidamente, promueven el equilibrio de género en la toma de decisiones, sin embargo, transcurridas ya más de dos décadas desde Beijing el déficit de participación de las mujeres en los procesos decisorios se mantiene en todos los ámbitos y niveles. En efecto, los estudios estadísticos tanto de las Naciones Unidas, como de la Unión Interparlamentaria, el Consejo de Europa y la Unión Europea, reflejan el lento proceso en la consecución de la participación equilibrada de género en los procesos decisorios, como muestran los datos publicados por las propias organizaciones internacionales.

Así, según las cifras publicadas por la Unión Interparlamentaria en la base de datos relativa a las "Mujeres en los parlamentos nacionales", a fecha de 1 de diciembre de 2018 las mujeres solo representan el $24.1 \%$ de los miembros de los Parlamentos a nivel mundial, habiendo más de 30 países en cuyos Parlamentos son mujeres menos del 10\% de sus miembros. En el desglose de los datos por regiones, se confirma que sólo los países nórdicos han alcanzado las exigencias de la representación equilibrada, con un $42.3 \%$ de mujeres en sus Parlamentos; en las Américas, las mujeres parlamentarias sólo llegan al 30.3\%; en Europa, si se excluyen los países nórdicos, sólo alcanzamos al $26.5 \%$, lo que significa que aproximadamente casi cuatro de cada cinco miembros de las cámaras parlamentarias son hombres; en el África subsahariana, la representación femenina en las instituciones legislativas se ha incrementado recientemente al 23.8\%; en Asia, aunque se ha mejorado la proporción de mujeres elegidas en los Parlamentos, solo alcanza al $19.7 \%$ de sus miembros; en los Países Árabes, las mujeres siguen estando muy infrarepresentadas, con tan sólo el $18.7 \%$ de los escaños parlamentarios; y en la región de las islas del Pacífico, las mujeres solo representan el $15.5 \%$ de los puestos en los Parlamentos (Inter-Parliamentary Union, 2018). Por lo que se refiere a los gobiernos y puestos ministeriales, sólo en 12 países las mujeres ocupan el 40\%, habiendo más de 10 países en cuyos gobiernos no hay una sola mujer.

Y en cuanto a las funciones más altas del Estado, las mujeres solo ejercen el $7.2 \%$ de las Jefatura de Estado y el 5.7\% de las Jefaturas de Gobierno (Inter-Parliamentary Union, 2017). Y en el seno de la propia Unión Interparlamentaria, aunque la propia organización ha modificado las normas estatutarias para establecer un sistema de cuota que asegure un umbral de participación equilibrada del $30 \%$ en la Asamblea Parlamentaria y el Consejo, y aunque la participación de las mujeres en las Asambleas se ha incrementado significativamente en los últimos años, sin embargo, en la 134 a Asamblea celebrada en Zambia en 2016 las mujeres solo representan el $29.9 \%$ de sus delegados (Inter-Parliamentary Union, 2016c). 
En el ámbito del Consejo de Europa, por lo que se refiere a la Asamblea Parlamentaria en 2016 no se había logrado todavía la participación equilibrada de género de al menos el 40\% de cada sexo, pues las mujeres solo representan el $36.6 \%$ de la composición de la cámara, ascendiendo solo a 21 las delegaciones estatales con igual o más del $40 \%$ de mujeres representantes, teniendo 16 delegaciones menos del $30 \%$ y habiendo incluso 4 delegaciones con menos del $20 \%$ de mujeres integrantes.

En el Tribunal Europeo de Derechos Humanos las mujeres representan de promedio solo el $35 \%$ de sus miembros (Council of Europe, 2017). Y por lo que se refiere a los Estados miembros, solo unos pocos cumplieron con el objetivo mínimo del $40 \%$ de ambos sexos elegidos en cualquiera de sus cuerpos legislativos en 2016, manteniéndose el promedio de mujeres elegidas en el 25\%. En los puestos más elevados del Estado, menos del 11\% de los países cumplieron con el objetivo mínimo del $40 \%$ para cada sexo, siendo el promedio de mujeres jefas de Estado o de gobierno, jefas de gobiernos regionales y alcaldesas inferior al $17 \%$. Y en cuanto al ámbito judicial, muy pocos tribunales alcanzaron el objetivo mínimo del 40\%, siendo la representación de mujeres en las Cortes Supremas del 33\%, en los Consejos Superiores del Poder Judicial del 28\% y en los Tribunales Constitucionales del 26\% (Council of Europe, 2017).

Y en el ámbito de la Unión Europea, a pesar de que la proporción de mujeres en las cámaras únicas/bajas de los Parlamentos nacionales de los Estados miembros ha aumentado del $22.1 \%$ en octubre de 2004 al $29.3 \%$ en noviembre de 2017, la tasa de progreso es lenta a poco más de medio punto porcentual por año. Si la tasa promedio de progreso no se recupera, se necesitará hasta 2050 para lograr la paridad de género. Por otro lado, existe una considerable variación entre los Estados miembros, pues en noviembre de 2017 los Parlamentos de Finlandia, Suecia y España incluyen al menos el 40\% de cada sexo, mientras que en 6 países (Chipre, Croacia, Grecia, Hungría, Letonia y Malta) las mujeres representan menos del $20 \%$ de los miembros.

Asimismo, aunque la proporción de mujeres en los puestos ministeriales es del $27.7 \%$, sin embargo, mientras que los gobiernos de Alemania, Dinamarca, Eslovenia, Francia y Suecia presentan un equilibrio de género, el de Hungría no incluye a ninguna mujer. Y a nivel regional, los datos muestran que las mujeres representan el $33.3 \%$ de los miembros de asambleas regionales en toda la Unión Europea, aunque las de Bélgica, España, Finlandia, Francia y Suecia incluyen al menos el $40 \%$ de cada género, pero las de Eslovaquia, Hungría, Italia y Rumanía integran menos del 20\% de mujeres (European Union, 2018a: 25-28). Por lo que se refiere al Parlamento Europeo, tras las elecciones de 2014 no se ha alcanzado la presencia equilibrada de al menos el $40 \%$ de personas del mismo sexo en la Eurocámara, pues el porcentaje de mujeres en el Parlamento Europeo al inicio de la legislatura de 2014-2019 solo alcanza al 35.8\%.

En su distribución por países, Malta es el país de la Unión que aporta mayor porcentaje de mujeres a la Eurocámara (67\%), le siguen Suecia e Irlanda (55\%), Finlandia (54\%) y Estonia (50\%). Del resto de países, solo Croacia (45\%), Austria (44\%), Francia y Países Bajos (42\%), Reino Unido y España $(41 \%)$ e Italia $(40 \%)$ alcanzan un porcentaje igual o superior al $40 \%$ de 
europarlamentarias. Los países que no alcanzan la masa crítica del $30 \%$ son Bélgica y Bulgaria (29\%), República Checa, Grecia y Polonia (24\%), Hungría (19\%) y Chipre (17\%), correspondiendo la menor proporción de mujeres a Lituania (9\%) (Unión Europea, 2014). En 2018, la proporción de mujeres en el Parlamento Europeo se ha incrementado mínimamente, alcanzado el 36.1\% (European Union, 2018b).

Por tanto, queda todavía mucho camino para hacer efectivo el principio de la participación equilibrada de al menos del $40 \%$ de mujeres y hombres en el seno de las propias organizaciones internacionales y en los Estados miembros. Como se ha analizado detenidamente, la consecución de la participación equilibrada de género en el ámbito internacional se ha llevado a cabo fundamentalmente mediante acuerdos, resoluciones y recomendaciones de escasa fuerza vinculante (soft law) que no siempre son aplicados por los Estados. Un buen ejemplo de esto es la resistencia de los Estados a adoptar acuerdos vinculantes que les obliguen a adoptar medidas internas de carácter legislativo que contribuyan a alcanzar la participación equilibrada de género, como refleja la propuesta de Directiva del Parlamento y del Consejo de la Unión Europea de 2012, que todavía no ha sido aprobada, por el rechazo de determinados Estados (Unión Europea, 2012b).

Sin embargo, atendiendo a la funcionalidad instrumental que presenta el principio de participación equilibrada de género en la toma de decisiones, pues, como se viene analizando, contribuye al pleno disfrute de los derechos humanos, constituye un elemento de justicia social y una condición necesaria para un mejor funcionamiento de la sociedad democrática, así como un presupuesto para alcanzar la efectiva igualdad de mujeres y hombres y evitar retrocesos en su consecución, contribuyendo a romper los estereotipos sexistas y a reducir la discriminación, es necesario que el principio de participación equilibrada de género y su plena consecución, la paridad de género, se reconozca e implemente a través de instrumentos jurídicos vinculantes en el marco internacional, tanto por las Naciones Unidas, como por la Unión Interparlamentaria, el Consejo de Europa y la Unión Europea, que obliguen a las propias organizaciones internacionales a su efectiva aplicación y a los Estados miembros a su reconocimiento y regulación con el máximo rango normativo, a nivel constitucional y legal, exigiendo la adopción de medidas eficaces que garanticen la consecución de la participación equilibrada de género en todos los procesos decisorios y a todos los niveles, especialmente en el ámbito político y público, incluyéndose mecanismos de seguimiento y sanción en caso de incumplimiento.

Y teniendo en cuenta que la población comprende aproximadamente la misma proporción de mujeres y hombres, la efectiva participación equilibrada de género se alcanzaría solo en los órganos en los que la mitad de sus miembros lo sean de cada sexo. Por lo tanto, para alcanzar una efectiva paridad de género en el ámbito político habrá que exigir que los gobiernos y los órganos electos, especialmente los Parlamentos, estén compuestos por la mitad de mujeres y la mitad de hombres. En esta línea, con el objetivo de lograr la paridad de género en el Parlamento Europeo tras las próximas elecciones que tienen lugar en mayo de 2019, el Lobby Europeo de Mujeres (EWL) ha presentado en junio de 2018 la campaña “50/50. Mujeres por Europa, Europa por las mujeres” (European 
Women's Lobby, 2018). Sin embargo, al tratarse de una campaña promocional y carecer de fuerza jurídica vinculante, es poco probable que se logre tal objetivo. Solo cuando la participación equilibrada y la paridad de género se reconozcan y regulen con eficacia normativa plena en el Reglamento interno de la Eurocámara y en los propios Tratados de la Unión Europea se logrará la efectiva paridad de género en el Parlamento Europeo.

\section{BibLiOgRAFÍA}

- Council of Europe (2003): "Recommendation Rec(2003)3 of the Committee of Ministers to member states on "Balanced participation of women and men in political and public decision making and explanatory memorandum", adopted by the Committee of Ministers on 12 March 2003 at the 831st meeting of the Ministers' Deputies. Disponible en: https://wcd.coe.int/ViewDoc.jsp?p=\&Ref=Rec(2003)3\&Language=lanEnglish\&Ver=original \&Bac kColorInternet=C3C3C3\&BackColorIntranet=EDB021\&BackColorLogged=F5D383\&direct=true [12/02/2019].

. (2004): "Resolution 1366 (2004) on "Candidates for the European Court of Human Rights", text adopted by the Parliamentary Assembly on 30 January 2004 (8th Sitting). Disponible en: http://assembly.coe.int/nw/xml/XRef/Xref-XML2HTML-en.asp?fileid=17194\&lang=en [12/02/2019].

. (2005): "Resolution 1426 (2005) on "Candidates for the European Court of Human Rights", adopted by the Parliamentary Assembly on 18 March 2005. Disponible en: http://assembly.coe.int/nw/xml/XRef/Xref-XML2HTML-en.asp?fileid=17311\&lang=en [12/02/2019].

. (2007): "Resolution 1585 (2007) on "Gender equality principles in the Parliamentary Assembly", text adopted by the Standing Committee, acting on behalf of the Parliamentary Assembly, on 23 November 2007. Disponible en: http://assembly.coe.int/nw/xml/XRef/XrefXML2HTML-en.asp?fileid=17604\&lang=en [12/02/2019].

. (2010): "Resolution 1706 (2010) on "Increasing women's representation in politics through the electoral system", text adopted by the Parliamentary Assembly on 27 January 2010 (6th Sitting). Disponible en: http://assembly.coe.int/nw/xml/XRef/Xref-XML2HTMLen.asp?fileid=17809\&lang=en [12/02/2019].

. (2011): "Resolution 1825 (2011) on "More women in economic and social decision-making bodies", text adopted by the Parliamentary Assembly on 23 June 2011 (26th Sitting). Disponible en: http://assembly.coe.int/nw/xml/XRef/Xref-XML2HTML-

en.asp?fileid=18014\&lang=en[12/02/2019]. 
. (2012): "Resolution 1898 (2012) on "Political parties and women's political representation", text adopted by the Parliamentary Assembly on 3 October 2012 (32nd Sitting). Disponible en: http://assembly.coe.int/nw/xml/XRef/Xref-XML2HTML-en.asp?fileid=19134\&lang=en [12/02/2019].

. (2013): “Gender Equality Strategy 2014-2017”, Gender Equality Commission (GEC), 6 November $2013 \quad[(\mathrm{CM}(2013) \quad 136$ final $] . \quad$ Disponible en: https://search.coe.int/cm/Pages/result_details.aspx?ObjectID=09000016805c7246 [12/02/2019].

. (2016a): "Resolution 2111 (2016) on "Assessing the impact of measures to improve women's political representation", text adopted by the Parliamentary Assembly on 21 April 2016 (16th Sitting). Disponible en: http://assembly.coe.int/nw/xml/XRef/Xref-XML2HTMLen.asp?fileid=22745\&lang=en [12/02/2019].

. (2016b): "Recommendation 390(2016) of the Congress of Local and Regional Authorities on "Women's political participation and representation at local and regional levels", adopted by the Congress on 20 October 2016, $2^{\text {nd }}$ Sitting. Disponible en: https://wcd.coe.int/com.instranet.InstraServlet?command=com.instranet.CmdBlobGet\&InstranetIma ge $=2946691 \&$ SecMode $=1 \&$ DocId=2388944 \&Usage $=2$ [12/02/2019].

. (2017): "Balanced participation of women and men in decision-making. Analytical report 2016 data". En: Gender Equality Commission (GEC). Disponible en: https://rm.coe.int/analyticalreport-data-2016-/1680751a3e\%20 [12/02/2019].

. (2018): “Gender Equality Strategy 2018-2023”, adopted by the Committee of Ministers on 7 March 2018. Disponible en: https://rm.coe.int/strategy-en-2018-2023/16807b58eb [12/02/2019].

- European Union (2014): "Resultados de las elecciones europeas de 2014. Distribución entre mujeres y hombres. Distribución por países". Parlamento Europeo. Disponible en: http://www.europarl.europa.eu/elections2014-results/es/gender-balance.html [12/02/2019].

. (2018a): "2018 Report on equality between women and men in the EU". European Commission. Luxembourg: Publications Office of the European Union. Disponible en: https://publications.europa.eu/en/publication-detail/-/publication/950dce57-6222-11e8-ab9c01aa75ed71a1 [12/02/2019].

. (2018b): "Women in the European Parliament". European Parliament. Disponible en: http://www.europarl.europa.eu/RegData/publications/2018/0001/P8_PUB\%282018\%290001_EN.p df [12/02/2019].

- European Women's Lobby (2018): “50/50: Women for Europe. Europe for Women”. Disponible en: https://www.womenlobby.org/Women-RE-shaping-Power-7741?lang=en [12/02/2019].

- Inter-Parliamentary Union (1997): "Specialized Inter-parliamentary Conference "Towards Partnership between Men and Women in Politics", Inter-parlamentary Union, New Delhi, India, 14-18 february. Disponible en: http://www.ipu.org/splz-e/ndelhi97.htm [12/02/2019]. 
(2011): "Inter-Parliamentary Union Strategy 2012-2017. Better Parliaments, Stronger Democracies", approved by the IPU Governing Council at its 189th session, Bern, 19 October, 2011. Disponible en: http://www.ipu.org/cnl-e/189/strategy.pdf [12/02/2019]. . (2012): "Plan of Action for Gender-sensitive Parliaments", adopted unanimously by the $127^{\text {th }} \quad I P U$ Assembly, Quebec, 26 October 2012. Disponible en: http://archive.ipu.org/pdf/publications/action-gender-e.pdf[12/02/2019].

(2016a): "Inter-Parliamentary Union Strategy 2017-2021. Strong democratic parliaments serving the people", approved by the IPU Governing Council at its 199th session, Geneva, 27 October, 2016. Disponible en: http://archive.ipu.org/cnl-e/199/strategy-17-21.pdf [12/02/2019]. (2016b): "The Freedom of women to participate in political processes fully, safely and without interference: Building partnerships between men and women to achieve this objective", Resolution adopted unanimously by the $135^{\text {th }}$ IPU Assembly, Geneva, 27 October 2016. Disponible en: http://www.ipu.org/conf-e/135/item4.pdf [12/02/2019].

(2016c): "Women in Delegations to Statutory sessiones". Disponible en: http://archive.ipu.org/wmn-e/dlgtns.htm [12/02/2019].

(2017): "Women in Politics: 2017. Situation on 1 January 2017". Disponible en: http://www.ipu.org/pdf/publications/wmnmap17-en.pdf [12/02/2019].

. (2018): "Women in National Parliaments. Situation as of 1St December 2018". Disponible en: http://archive.ipu.org/wmn-e/world.htm [12/02/2019].

- Moss Kanter, R. (1977): "Some effects of proportions on group life: Skewed sex ratios and responses to token women”. En: American Journal of Sociology, vol. 82, núm. 5, pp. 965-990.

- Naciones Unidas (1996): “Informe de la Cuarta Conferencia Mundial sobre la Mujer”, Beijing, 4 a 15 de septiembre de 1995. Nueva York: Naciones Unidas. Disponible en: http://www.un.org/womenwatch/daw/beijing/pdf/Beijing\%20full\%20report\%20S.pdf [12/02/2019]. . (1997): "Recomendación General No 23, sobre la "Vida Política y Pública", adoptada por el Comité para la Eliminación de la Discriminación contra la Mujer, $16^{\circ}$ Período de sesiones, Naciones Unidas (Doc. A/52/38). Disponible http://www.un.org/womenwatch/daw/cedaw/recommendations/recomm-sp.htm [12/02/2019].

. (2000): “Informe del Comité Especial Plenario del vigésimo tercer período extraordinario de sesiones de la Asamblea General", Nueva York, Naciones Unidas, Suplemento No. 3 (A/S23/10/Rev.1). Disponible en: http://www.un.org/ga/search/view_doc.asp?symbol=A/S 23/10/REV.1\&referer=https://www.google.es/\&Lang=S [12/02/2019].

. (2006): "Informe sobre el 50 periodo de sesiones (22 de marzo de 2005, 27 de febrero a 10 de marzo y 16 de marzo de 2006)", Comisión de la Condición Jurídica y Social de la Mujer, Consejo Económico y Social, Documentos Oficiales, Suplemento No. 7. Nueva York: Naciones Unidas (E/2006/27-E/CN.6/2006/15). Disponible, en: https://documents-ddsny.un.org/doc/UNDOC/GEN/N06/295/21/PDF/N0629521.pdf?OpenElement [12/02/2019]. 
. (2007): "Resolución A/RES/62/137de la Asamblea General de las Naciones Unidas sobre el "Seguimiento de la Cuarta Conferencia Mundial sobre la Mujer y plena aplicación de la Declaración y la Plataforma de Acción de Beijing y de los resultados del vigésimo tercer período extraordinario de sesiones de la Asamblea General", 18 de diciembre de 2007. Disponible en: http://undocs.org/es/A/RES/62/137 [12/02/2019].

(2015): "Resolución A/RES/70/1 de la Asamblea General relativa a "Transformar nuestro mundo: la Agenda 2030 para el Desarrollo Sostenible", de 25 de septiembre de 2015. Disponible en: http://www.un.org/es/comun/docs/index.asp?symbol=A/RES/70/1\&referer=http://www.un.org/es/g a/70/resolutions.shtml\&Lang=S [12/02/2019].

- Saldaña Díaz, María Nieves (2006): “Aproximación al concepto de ciudadanía europea desde la perspectiva de género: el presupuesto político de la transversalidad”. En: Francisco Javier Pérez Royo, et al. (coords.): Derecho Constitucional para el siglo XXI. 2 vols. Navarra: Editorial Aranzadi, vol. 1, pp. 731-758.

. (2010): "Derechos humanos y democracia paritaria en las Naciones Unidas: un reto por conquistar en el Siglo XXI”. En: Juán Jesús Mora Molina (coord.): Jaque a la Democracia. Madrid: Editorial Sepha, pp. 75-110.

. (2013): "Génesis y promoción de la democracia paritaria en el Consejo de Europa: de la igualdad entre mujeres y hombres como fundamento de la democracia a la participación equilibrada de género en la toma de decisiones política y pública”. En: Revista Europea de Derechos Fundamentales, $\mathrm{n}^{\mathrm{o}}$. 22, pp. 137-204.

. (2014): “La acción de la Unión Interparlamentaria en defensa de la igual participación de las mujeres en los parlamentos: alianza de géneros en la esfera política, participación equilibrada de género y parlamentos sensibles al género". En: Igualdad y democracia: el género como categoría de análisis jurídico. Estudios en homenaje a la Profesora Julia Sevilla Merino. Valencia: Corts Valencianes, pp. 541-562. Disponible en: http://rabida.uhu.es/dspace/handle/10272/8385 [12/02/2019].

- Unión Europea (1996): "Recomendación del Consejo 96/694/E, de 2 de diciembre de 1996, relativa a la "Participación equilibrada de las mujeres y los hombres en los procesos de toma de decisión”, DO L 319, 10.12.1996, pp. 11-15. Disponible en: http://eur-lex.europa.eu/legalcontent/ES/TXT/?uri=CELEX\%3A31996H0694 [12/02/2019].

(1999): "Communication from the Commission "Women and Science, Mobilising women to enrich European research", Brussels, COM(1999) 76 Final, OJ L 154, 27.06.2000, pp. 34-35. Disponible en: http://aei.pitt.edu/13321/1/13321.pdf [12/02/2019].

. (2000a): “Comunicación de la Comisión al Consejo, al Parlamento europeo, al Comité económico y social y al Comité de las Regiones, "Hacia una estrategia marco comunitaria sobre la igualdad entre hombres y mujeres (2001-2005)", Bruselas, 7.6.2000 $\operatorname{COM(2000)~} 335$ final. 
Disponible

en:

http://www.empleo.gob.es/uafse_2000-

2006/equal/descargas/estrategia\%20marco\%20comunitaria\%20igualdad.pdf [12/02/2019].

(2000b): "Decisión de la Comisión, de 19 de junio de 2000, relativa al "Equilibrio entre hombres y mujeres en los Comités y Grupos de Expertos creados por la Comisión", DOCE L 154/34, 27.06.2000, pp. 34-35. Disponible en: http://eur-lex.europa.eu/legalcontent/ES/TXT/PDF/?uri=CELEX:32000D0407\&from=ES [12/02/2019].

. (2001): "Resolución del Parlamento Europeo sobre el "Informe de la Comisión sobre la aplicación de la Recomendación 96/694 del Consejo de 2 de diciembre de 1996, relativa a la participación equilibrada de las mujeres y los hombres en los procesos de toma de decisión”, DOCE C 262, 18.9.2001, pp. 248-251 [COM(2000)120-C5-0210/2000- 2000/2117(COS)]. Disponible en: http://www.europarl.europa.eu/sides/getDoc.do?pubRef=-//EP//TEXT+TA+P5-TA-20010034+0+DOC+XML+V0//ES [12/02/2019].

. (2008): "Resolución del Parlamento Europeo, de 21 de mayo de 2008, sobre "La mujer y la ciencia". Disponible en: http://www.europarl.europa.eu/sides/getDoc.do?pubRef=//EP//TEXT+TA+P6-TA-2008-0221+0+DOC+XML+V0//ES [12/02/2019].

(2012): "Propuesta de Directiva del Parlamento Europeo y del Consejo destinada a mejorar el equilibrio de género entre los administradores no ejecutivos de las empresas cotizadas y por la que se establecen medidas afines", Bruselas, 14.11.2012 [COM(2012) 614 final]. Disponible en: http://eur-lex.europa.eu/legal-

content/ES/TXT/PDF/?uri=CELEX:52012PC0614\&from=EN[12/02/2019].

. (2012a): "Resolución del Parlamento Europeo, de 13 de marzo de 2012, relativa a "La representación de las mujeres en los procesos de toma de decisiones políticas: calidad e igualdad", DO C 251E, 31.08.2013, pp. 11-18. Disponible en: http://www.europarl.europa.eu/sides/getDoc.do?pubRef=-//EP//TEXT+TA+P7-TA-20120070+0+DOC+XML+V0//ES [12/02/2019].

. (2016): “Compromiso Estratégico para la Igualdad entre Mujeres y Hombres 2016-2019”, aprobado por la Comisión Europea el 3 de diciembre de 2015. Luxemburgo: Oficina de Publicaciones de la Unión Europea. Disponible en: http://ec.europa.eu/justice/genderequality/document/files/strategic_engagement_es.pdf [12/02/2019].

. (2019): "Resolución del Parlamento Europeo, de 15 de enero de 2019, relativa a "La integración de la perspectiva de género en el Parlamento Europeo". Disponible (texto e inglés) en: http://www.europarl.europa.eu/sides/getDoc.do?pubRef=-//EP//NONSGML+TA+P8-TA-20190010+0+DOC+PDF+V0//EN [12/02/2019]. 